\title{
A MANUAL FOR AUTHORS OF MATHEMATICAL PAPERS*
}

The purpose of this manual is to help mathematicians in preparing papers for publication. A reasonable amount of attention on the part of authors to the rules and suggestions given below will save the time of editors, referees, printers, and readers, and will help keep the cost of publication at a minimum. Although most of the discussion will apply to mathematical papers in general, special rules will occasionally be given for papers specifically intended for the Bulletin or Transactions of the American Mathematical Society.

Style. In the course of writing a mathematical paper intended for publication, the author must settle such questions of usage and arrangement as (1) the organization of the paper into chapters, sections, paragraphs, propositions, theorems, proofs, examples, remarks, and the use of appropriate headings; (2) the adoption of a system of enumeration for the sections, theorems; (3) the treatment of footnotes, references and bibliography; (4) the use of capital or small initial letters in such adjectives as abelian, euclidean, jacobian; (5) the use or non-use of hyphens in such technical terms as sub-harmonic, fixed-point; (6) the questions of style which arise when words in the text are replaced by symbols.

Concerning the first three questions-particularly the third-the author's decisions must to some extent be governed by the routine stylistic requirements of the journal for which his paper is intended. For guidance, the author should consult current numbers of that journal; if his paper is intended for the Bulletin or Transactions, he may consult, instead, the Special Rules given below.

Concerning the remaining questions, perhaps the only general rule that can be offered is the Rule of Uniformity: Whatever the usage adopted by an author in a given paper, that usage should be maintained consistently throughout the paper. It may be remarked that the reasons for urging a careful observance of this rule are economic as well as stylistic. Every paper is carefully checked by an editorial assistant before it is sent to the printer. An author who has written "euclidean" part of the time and "Euclidean" the rest of the time may have done so inadvertently. But the assistant cannot guess this.

* The writers of this manual have received invaluable advice from members of the office staff of the American Mathematical Society and have consulted freely the following excellent pamphlets: Notes on the preparation of mathematical papers (The London Mathematical Society); Typographic suggestions to authors (George Banta Publishing Co.); Author's manual (Duke Mathematical Journal). 
Nor can one guess whether the author prefers the capital or the small letters. The correspondence between author and editorial staff which must take place in order to settle the matter causes delay and expense which could easily have been avoided.

The use of mathematical symbols in the solid text is not only permissible but is frequently encouraged by editors as a means for securing greater economy of space on the printed page. Authors, however, should consider carefully the questions of style which arise from this practice, particularly in the use of " $<$ " and " =". Such phrases as "assume $x>y$ " and "assume that $x$ is $>y$ " are in common use and there appears to be no widely accepted ground for preferring one to the other. On the other hand, no one would write "assume that $x$ and $y$ are $="$ although the objection appears to be largely visual. Some writers do not hesitate to begin a sentence with a mathematical symbol, but others regard this as a barbarism. ${ }^{1}$ In the complete absence of agreement on these questions, we offer only the following general rules in addition to the Rule of Uniformity:

1. Symbols in the text should be used in such a way that their translation into words or phrases will require no effort on the part of the reader.

2. The resulting translation should conform to the standards of good literary composition.

Rules 1 and 2 are admittedly not decisive in all cases. Thus "let $\epsilon \geqq 0$ be a number such that ..." would be objectionable on both counts, but is probably preferable, in appearance at least, to "let $\epsilon$ be a number $\geqq 0$ such that ... ." One could, of course, avoid both forms by writing "let $\epsilon$ be a non-negative real number ...." Again, "...the expression on the left is < the smallest value of ..." satisfies rules 1 and 2 . Yet the substitution of two words for " $<$ " would result in a far better appearance. This improvement is surely worth the slight extra effort involved.

The printing of mathematical symbols. There are unfortunately many useful arrangements of symbols which must be avoided in a manuscript intended for publication, on account of the difficulties which would have to be overcome by the compositor. A type consists of a solid block or "body" with a raised character on one of its rectangular faces. The bodies are normally laid side by side by a machine and form a row of uniform width. Besides the ordinary

1 There is a similar division of opinion concerning the use of the "hanging theorem" in which the word THEOREM is simultaneously the first word of a paragraph and the last word of the sentence which precedes it. 
alphabets, most printers have on hand a number of "dashed letters" $(\bar{A}, \bar{a}, \cdots)$ and "tilde letters" $(\tilde{A}, \cdots)$. If a dashed letter, say $\overline{\mathfrak{a}}$, is not in the printer's possession, it does not follow that this letter cannot be printed. But the typesetting involved is much more complicated and includes a number of operations by hand. If $\mathfrak{a}$ occurs in the solid text, the body of an ordinary $a$ must be reduced in size by filing, to make room for a thin body carrying the bar. The two bodies must then be placed in position by hand. The thin body may or may not stay in place and thus the alignment of the bar may or may not be correct. Moreover, the bars, when printed, may not always be uniform. Thus to the extra expense involved in the formation and typesetting of the symbol $\overline{\mathfrak{a}}$, it is necessary to add the cost of re-alignment or re-setting in case of mishaps during the printing of proof.

The typesetting of inferior and superior symbols (subscripts and superscripts) in the solid text offers no difficulty when those symbols are available as inferiors and superiors (see Appendix C) and when no symbol is to be placed directly above or below another. Thus, $(a+b x)^{n / 3}, A^{2}$ can be set by machine, but $x^{\frac{n}{3}}, A_{3}^{2}$ require hand work. ${ }^{2}$ Subscripts to superscripts and superscripts to subscripts, all belonging to a single term, are extremely difficult to set, even by hand.

In displayed formulas, the insertion of bars, subscripts and the like is somewhat less difficult since there is space above and below, whereas in the solid text, the compositor has only a single line with which to work. Thus the symbol $A_{3}^{2}$ no longer requires hand work since the superscript 2 can be set by machine in the subscript position of the line above the line of the $A$. Note however that when set in this manner, the superscript will be slightly higher: $A_{3}^{2}$ rather than $A_{3}^{2}$.

Special rules. Authors of papers intended for the Bulletin or Transactions should give special attention to the following rules. A reasonably strict observance of these rules will help keep printing costs down, prevent an uneconomical use of space, insure a certain uniformity of appearance, and will greatly simplify the task of final preparation of manuscripts for the printer.

1. Use only such characters as the printer has in stock. The list in Appendix $\mathrm{C}$ includes all special types now available for the Bulletin and Transactions. Additions to this list are made from time to time. Note particularly what letters and numbers are available in sub-superscript (inferior to superior) and super-subscript positions.

${ }^{2}$ These remarks are not intended to imply that symbols such as $A_{3}^{2}$ must never be used in the solid text, rather that the author should exercise a measure of discretion. 
Note also that many symbols are not available in footnote size. For this reason, if for no other, complicated formulas should not be put into the footnotes. In this connection it might be remarked that an excessive number of footnotes sometimes gives the undesirable impression that the paper is being "written in the footnotes." Careful organization, however, always makes it possible to reduce the number and length of footnotes, even to eliminate them entirely.

2. Do not put into the solid text any formula which would require spreading (uneven spacing) of lines.

3. In the solid text, write $\sum_{i=1}^{n} ; \prod_{i=1}^{n} ; \lim _{n \rightarrow \infty} ; \int_{a}^{b}$. In the first two examples the $n$ should be directly over the $i$. In the integral the $b$ should be directly over the $a$; it is not feasible to place the $a$ nearer the integral sign since the latter is on a rectangular face:[]. In displayed formulas, the limits should occupy only one line. For example, write

$$
\sum_{i=i_{0}, j=j_{0}}^{\infty} \text { instead of } \sum_{\substack{i=i_{0} \\ j=j_{0}}}^{\infty} \text {. }
$$

4. In the text, replace $e^{()}$by exp ( ) if the expression in the parentheses is complicated.

5. Use $C_{m, n}$ (binomial coefficient) instead of $\left(\begin{array}{l}m \\ n\end{array}\right)$.

6. In the text, write $\sim_{\epsilon}$, in display $\sim$.

7. Use $\in$ to mean "is a member of," $\epsilon$ for epsilon. The symbol for "is not a member of" is $\notin$.

8. Use fractional exponents in place of radicals. A fraction occurring as subscript or superscript should be formed with a solidus, for example $2 / 3$. In display, the number of lines occupied by a fraction should be as small as possible. Frequently the number of lines can be reduced by a simple rearrangement. For example:

$$
\frac{\cos \frac{1}{x}}{\sqrt{a+\frac{b}{x}}} \text { can be written } \quad \frac{\cos (1 / x)}{(a+b / x)^{1 / 2}} .
$$

9. Long formulas, or expressions which might break awkwardly at the end of a line of text, should be displayed.

10. In numbering sections, theorems, displayed formulas, and so on, the author should adopt a system which is simple and selfexplanatory. A system based on such symbols as II.3.6.1a is unnecessarily complicated. 
11. Footnotes should be assembled at the end of the paper, typed double spaced, and numbered with arabic numbers. The footnote symbols currently in use are illustrated as follows: "It was proved ${ }^{3}$ that ..." (Bulletin); "It was proved $\left(^{3}\right)$ that . . ." (Transactions). A footnote symbol should not follow a mathematical symbol. Footnotes should be numbered in the order in which they appear, beginning with 1 and continuing throughout the paper. Do not use any symbol at all for the footnote "Presented to ..." which frequently appears on the title page.

12. Mathematical works which are referred to in the text may be listed at the end of the paper under the heading "Bibliography" or "References." The following examples illustrate the two methods of arrangement and enumeration used in the Bulletin and Transactions:

\section{Form I \\ Bibliography [or RefERENCES]}

1. R. R. Roe, Canonical systems, Amer. J. Math. vol. 60 (1938) pp. 1042-1100.

2. - An elementary solution of the fundamental problem of mathematics, J. of Metamath. vol. 17 (1939) pp. 1-100.

3. D. D. Doe, The theory of topological hypergroups, New York, 1940.

R. R. RoE

\section{Form II \\ Bibliography [or ReFERENCES]}

1. Canonical systems, Amer. J. Math. vol. 60 (1938) pp. 1042-1100.

2. An elementary solution of the fundamental problem of mathematics, J. of Metamath. vol. 17 (1939) pp. 1-100.

D. D. DoE

1. The theory of topological hypergroups, New York, 1940.

2. A generalization of the generalized Doe-differential, Duke Math. J. vol. 48 (1940) pp. $1100-1200$.

The following examples illustrate the various methods of referring to the bibliography. The basic marks of enclosure are brackets: [ ]. With a small amount of care, the use of parentheses as additional marks of enclosure can be kept at a minimum and the appearance of the printed page thereby improved.

For form I: [1, Theorem 3.7]; $\cdots$ proved by Roe [2, Lemma 4] $\cdots$; (see [2, Lemma 4]).

For form II: [Roe 1, Theorem 3.7]; $\cdots$ proved by Roe [2, p. 100] $\cdots$; (cf. Roe [2, p. 100]).

The use of abbreviations for the names of the more obscure scientific journals often creates difficulties for readers and librarians. The Bulletin and Transactions, therefore, make it a rule to print such 
titles without any abbreviations. Cooperation on the part of the authors would diminish greatly the work involved in this connection. The names of the standard journals, however, should be abbreviated. Appendix B contains a list of abbreviations which have been adopted by the Bulletin and Transactions.

Preparation of the manuscript. A compositor is not a trained mathematician and cannot take the responsibility of altering so much as the position of a single dot in the manuscript. It is obvious, therefore, that the manuscript, when it is sent to the printer, must be exact in every detail. Since it is impossible to produce a manuscript in which all characters are facsimiles of printed characters, there will be many details which can be made clear to the printer only by special indication such as underlining or marginal notes.

The task of preparing the manuscript for the printer is generally shared between author and editorial staff. In the case of the Bulletin and Transactions, indications concerning routine matters of style, such as the spacing of material, the choice of type in titles, paragraph headings, and so on, are inserted by an editorial assistant. All indications of a non-routine nature must be made by the author and must be complete and explicit. The most important rules in this connection are the following: ${ }^{3}$

1. The manuscript should be typewritten. The text should be double spaced and displayed material should not be crowded. Plenty of space should be left for the characters which are to be filled in by hand. It is well to make at least one carbon copy of the manuscript, but the original-not the carbon copy-should be sent to the editor.

2. Subscripts or superscripts may be filled in by hand; if typed they should be a half-space above or below the line.

3. Handwritten characters should be made with great care and each character must be distinct in shape from every other. In cases where the author's intention may be in doubt, identification, such as "Greek chi," should be written in pencil in the margin. Capital letters may be indicated by "cap," small letters by "1.c." (lower case). Letters and symbols which are particularly liable to confusion are:

$$
\begin{gathered}
o O 0 \quad \gamma r n \nu \quad \rho p \quad s S 5 \quad 1, \quad 1 \prime \quad h k \kappa K \mathrm{~K} \\
z Z 2 \quad \psi \times x \times 4 \quad \text { el } 1 \quad V \cup \quad \in \epsilon
\end{gathered}
$$

4. German and script letters may be handwritten if they can be made with sufficient accuracy. A far more satisfactory procedure is to type the corresponding Roman letters, then to underline or encircle

${ }^{3}$ See also rules 3-8 in the section Special Rules. 
each letter with colored pencil, say green for German, blue for script. In fact, all alphabets other than the Roman and Greek are best treated in this manner. The meaning of the colored marks should be stated at appropriate places in the margin or on a separate sheet of "instructions for the printer."

5. No mark meaning "Greek alphabet" should be attached to the symbols $\sum$ (summation), $\Pi$ (product), $\partial$ (in partial derivatives). Notice the difference in the size and style between the first two symbols and the Greek capital letters $\Sigma, \Pi$.

6. Italic type is indicated by underlining, preferably with a blue pencil. In the Bulletin and Transactions, the text of a theorem or corollary is always in italics and the necessary indications may be left to an editorial assistant.

7. Omissions should be denoted by exactly three slightly raised dots, such as $a b \cdots c$. If commas are required, write $a_{1}, \cdots, a_{n}$ or $a_{1}, a_{2}, \cdots$, never $a_{1}, \cdots a_{n}$ or $a_{1}, a_{2} \cdots$. A handwritten comma must be carefully made so that it will not be taken for subscript 1 .

8. The number one is ordinarily typed 1 . The letter "ell" is denoted by 1 (the dash may be written). Experience shows that the use of the solidus (/) in place of the dash may lead to errors in typesetting.

9. Drawings and diagrams should be made with particular care since they cannot be changed or corrected in galley proofs or page proofs. Drawings should be made in black India ink on Bristol board and should be two or three times larger than they are to be when printed. When inserting letters or symbols, allowance should be made for the reduction in size. For drawings which are to appear in the Bulletin or Transactions, all letters and symbols should be drawn lightly in pencil since they will eventually be replaced by type letters and symbols. The reproduction of even the simplest drawing involves considerable extra expense, and the author, therefore, should include no drawing which is not really essential for a clear understanding of his paper.

10. A displayed formula should be numbered only if referred to elsewhere in the text. If displayed formulas are numbered indiscriminately, it is impossible to combine several formulas into a single line or to run an occasional formula into the text to facilitate the breaking up of galleys into pages.

Proof reading. It is important that galley proofs be carefully read and corrected by the author, since it is only the author who can detect errors which are due to an imperfect manuscript.

Alterations in type which has been set are delicate and costly to 
make. The author should call for only such changes as are really essential. The insertion or deletion of a comma, for example, should be based on a compelling reason and should not be merely a matter of whim. If a number of consecutive words or symbols must be deleted, they should if possible be replaced by words or symbols occupying the same amount of space. If this is not done, the compositor often must tear down and reset a number of preceding or following lines in order to make the necessary adjustments. This is not only costly but makes possible the introduction of new errors.

Galley proofs of papers for the Bulletin or Transactions are read in the office of the American Mathematical Society before they are sent to the author. During the reading, there may arise questions which can be answered only by the author. Such questions are written in the margins, and it would seem unnecessary to point out that they should be answered were it not for the fact that they are frequently ignored.

The list in Appendix A contains most of the standard signs used in this country in correcting proof. A consistent use of these signs by authors would simplify greatly the work of printer and editorial staff. In any case, authors should make sure that their corrections are clear and explicit and should refrain from using elaborate "systems" of their own invention. Joint authors should be particularly careful not to use two non-equivalent systems of signs in a single set of galley.

The example in Appendix A illustrates the standard method of using the proof reading signs. It is important to note that all indic . tions for corrections must appear in margins and for each correction a corresponding mark must appear in the text. The signs in the margins should retain the order of the corresponding errors in the line and should be separated from each other by $\mid$. 


\begin{tabular}{|c|c|}
\hline \multicolumn{2}{|c|}{$\begin{array}{c}\text { APPENDIX A } \\
\text { SIGNS USED IN CORRECTING PROOFS }\end{array}$} \\
\hline$\ell$ Delete; take out & $x$ Change broken letter \\
\hline$\bigodot$ Close up & stet Let it stand as set \\
\hline$\wedge$ Insert & $\cdots \cdots$ Let it stand as set \\
\hline \# Insert space & w.f Wrong font, size or style \\
\hline$\neg$ Raise & l.c. Lower case, not capitals \\
\hline$\sqcup$ Lower & hom. Use Roman letter \\
\hline [ Move to left & bf. Use black type letters \\
\hline ] Move to right & $\odot$ Period \\
\hline $\begin{array}{l}\| \text { Straighten type line at side } \\
\text { of page }\end{array}$ & ^ Comma \\
\hline$/ /$ Straighten lines & ¿ Apostrophe \\
\hline 历 Paragraph & $2^{2}$ Superior figure \\
\hline center Put in middle of page or & - $\hat{2}$ Inferior figure \\
\hline & $=/$ Hyphen \\
\hline$\circlearrowleft$ Transpose & sc. Use small capitals \\
\hline $\begin{array}{l}\text { Th Transpose } \\
\text { T Turn inverted letter right } \\
\text { side up }\end{array}$ & Cops Use capitals \\
\hline
\end{tabular}




\section{EXAMPLE SHOWING THE USE OF PROOF READING SIGNS}

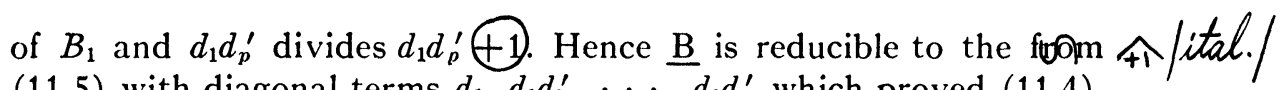
(11.5) with diagonal terms $d_{1}, d_{1} d_{2}^{\prime}, \ldots, d_{1} d_{\rho}^{\prime}$ which proved (11.4).

Bf. 12. Groups with (a)finite number of generators. We shall discuss certain properties of these groups culminating in the basic product $p / \sqcap$ decomosition (12.5).

(12.1) Definition Let $B=\left\{g_{1}, \cdots, g_{n}\right\}, B^{\prime}=\left\{g_{1}^{\prime}, \cdots \cdot g_{n}^{\prime}\right\}$ be A.C./ $/ \lambda$

\# two sets of elementspof $G$ containing the same number $n$ of elements. By

h a unimodulat transformation $\tau: B \rightarrow B^{\prime}$ is meant a system of $f_{\wedge}$ elations $h=$

$$
g_{i}^{\prime}=\sum a_{i j} g_{j}, \quad\left\|a_{i j}\right\| \text { unimodular. }
$$

\$ The following proposition shows in how natural a manner unimodular transformations make their appearance in the theory of gro@ps with rom finite bases. (12.3) Let $G$ be a group with a finite base $B=\left\{g_{1}, \cdots, g_{n \wedge}\right.$. In order $\}$
rimeV that $B^{\prime}=\left\{g_{1}^{\prime}, \cdots, g_{n}^{\vee}\right\}$ be a base for $G$ it is necessary and sufficient that $B^{\prime}$ be oblainable from $B$ by a unimodula $\widehat{r}$ transformation.

For any given set $B^{\prime}=\left\{g_{1}^{\prime}, \cdots, g_{n}^{\prime}\right\}$ of elements of $G$ there exist relations

nimeV (12.4)

$$
g_{i}^{\check{Y}}=\sum c_{i j} g_{j}, \quad C=\left\|c_{i j}\right\|_{\wedge}
$$

A necessary and sufficient condition order that $\left\{g_{i}^{\prime}\right\}$ be as base is $M / B^{\prime}=/ N$

l.c. that the $\phi_{j}$ be expressible as linear combinations of the $g_{i}^{\prime}$, or that there exist relations

$$
] g_{i}=\sum d_{i j} g_{j}^{\prime}, \quad D=\left\|d_{i j}\right\| \cdot[
$$

center

$[\quad$ From this follows

$$
g_{i}=\sum d_{i j} c_{j k} g_{k j}
$$

Hence since $B$ is a base we must have $D C=1$.)

This matrix relation yields $|D| \cap|C|=1$, and since the deter-hun in minants are integers we must have $|C|= \pm 1$. Thus in order that $B^{\prime}$ be a base $C$ must be unimodular, or the condition of (12.3) must be

[ [ fulfilled. Conversely, if $j t$ is fulfilled, $C$ is unimodular and (12.5) (12.3) holds with $D=C \in 1$, from which it follows readily that $\underline{\underline{b^{\prime}}}$ is a base. $\forall / c a p$ 


\section{THE PRECEDING PASSAGE PRINTED WITH ALL CORRECTIONS MADE}

of $B_{1}$ and $d_{1} d_{p}^{\prime}$ divides $d_{1} d_{p+1}^{\prime}$. Hence $B$ is reducible to the form (11.5) with diagonal terms $d_{1}, d_{1} d_{2}^{\prime}, \cdots, d_{1} d_{\rho}^{\prime}$ which proves (11.4).

12. Groups with a finite number of generators. We shall discuss certain properties of these groups culminating in the basic product decomposition (12.5).

(12.1) Definition. Let $B=\left\{g_{1}, \cdots, g_{n}\right\}, B^{\prime}=\left\{g_{1}^{\prime}, \cdots, g_{n}^{\prime}\right\}$ be two sets of elements of $G$ containing the same number $n$ of elements. $B y$ a unimodular transformation $\tau: B \rightarrow B^{\prime}$ is meant a system of relations

$$
g_{i}^{\prime}=\sum a_{i j} g_{j}, \quad\left\|a_{i j}\right\| \text { unimodular. }
$$

The following proposition shows in how natural a manner unimodular transformations make their appearance in the theory of groups with finite bases.

(12.3) Let $G$ be a group with a finite base $B=\left\{g_{1}, \cdots, g_{n}\right\}$. In order that $B^{\prime}=\left\{g_{1}^{\prime}, \cdots, g_{n}^{\prime}\right\}$ be a base for $G$ it is necessary and sufficient that $B^{\prime}$ be obtainable from $B$ by a unimodular transformation.

For any given set $B^{\prime}=\left\{g_{1}^{\prime}, \cdots, g_{n}^{\prime}\right\}$ of elements of $G$ there exist relations.

$$
g_{i}^{\prime}=\sum c_{i j} g_{j}, \quad C=\left\|c_{i j}\right\| .
$$

A necessary and sufficient condition that $B^{\prime}=\left\{g_{i}^{\prime}\right\}$ be a base is that the $g_{j}$ be expressible as linear combinations of the $g_{i}^{\prime}$, or that there exist relations

$$
g_{i}=\sum d_{i j} g_{j}^{\prime}, \quad D=\left\|d_{i j}\right\| .
$$

From this follows

$$
g_{i}=\sum d_{i j} c_{j k} g_{k}
$$

Hence since $B$ is a base we must have $D C=1$. This matrix relation yields $|D| \cdot|C|=1$, and since the determinants are integers we must have $|C|= \pm 1$. Thus in order that $B^{\prime}$ be a base $C$ must be unimodular, or the condition of (12.3) must be fulfilled. Conversely, if (12.3) is fulfilled, $C$ is unimodular and (12.5) holds with $D=C^{-1}$, from which it follows readily that $B^{\prime}$ is a base. 


\section{APPENDIX B \\ ABBREVIATIONS USED IN
THE BULLETIN AND TRANSACTIONS}

Abhandlungen aus dem Mathematischen Se- Abh. Math. Sem. Hansischen Univ. minar der Hansischen Universität (formerly ... Hamburgischen Universität)

Academy of Sciences of the USSR. Journal of Acad. Sci. USSR. J. Phys. Physics

Acta Universitatis Szegediensis. Acta Scientia- Acta Univ. Szeged. rum Mathematicarum

Acta Mathematica

American Journal of Mathematics

The American Mathematical Monthly

American Mathematical Society Colloquium Publications

American Statistical Association Bulletin

Annales de la Faculté des Sciences de l'Université de Toulouse pour les Sciences Mathématiques et les Sciences Physiques

Annales Scientifiques de l'École Normale Supérieure

The Annals of Mathematical Statistics

Annals of Mathematics

Atti della Reale Accademia delle Scienze di Torino. Classe di Scienze fisiche, Matematiche e Naturali

Il Bollettino di Matematica

Bulletin de l'Académie des Sciences de l'URSS. Série Mathématique

Bulletin de la Société Mathématique de France

Bulletin de la Société Physico-Mathématique de Kazan et de l'Institut de Mathématiques et Mécanique

Bulletin des Sciences Mathématiques

Bulletin Mathématiques de l'Université de Moscou. Série Internationale

Bulletin of the American Mathematical Society

Bulletin of the Calcutta Mathematical Society

Commentarii Mathematici Helvetici

Compositio Mathematica

Comptes Rendus (Doklady) de l'Académie des Sciences de l'URSS

Comptes Rendus Hebdomadaires des Séances de l'Académie des Sciences

Doklady Akademii Nauk SSSR

See Comptes Rendus (Doklady) de l'Académie des Sciences de l'URSS

Duke Mathematical Journal

Fundamenta Mathematicae

Acta Math.

Amer. J. Math.

Amer. Math. Monthly

Amer. Math. Soc. Colloquium Publications

Amer. Statist. Assoc. Bull.

Ann. Fac. Sci. Univ. Toulouse

Ann. École Norm.

Ann. Math. Statist.

Ann. of Math.

Atti Accad. Sci. Torino

Boll. Mat.

Bull. Acad. Sci. URSS. Sér. Math.

Bull. Soc. Math. France

Bull. Soc. Phys.-Math. Kazan

Bull. Sci. Math.

Bull. Math. Univ. Moscou

Bull. Amer. Math. Soc.

Bull. Calcutta Math. Soc.

Comment. Math. Helv.

Compositio Math.

C. R. (Doklady) Acad. Sci. URSS.

C. R. Acad. Sci. Paris

Duke Math. J.

Fund. Math.

Izvestia Akademii Nauk SSSR

See Bulletin de l'Académie des Sciences de l'URSS 
Jahresbericht der Deutschen Mathematiker Jber. Deutschen Math. Verein. Vereinigung

Japanese Journal of Mathematics. Transactions Jap. J. Math. and Abstracts

Journal de l'École Polytechnique

Journal de Mathématiques Pures et Appliquées

Journal für die reine und angewandte Mathematik

The Journal of Symbolic Logic

Journal of the Faculty of Science. Imperial University of Tokyo. Section I. Mathematics, Astronomy, Physics, Chemistry

The Journal of the Indian Mathematical Society

The Journal of the London Mathematical Society

Koninklijke Nederlandsche Akademie van Wetenschappen. Indagationes Mathematicae ex Actis Quibus Titulus. Proceedings of the Section of Sciences (sometimes referred to as the "Amsterdam Proceedings")

Matematičeskij Sbornik

See Recueil Mathématique

Mathematische Annalen

Mathematische Zeitschrift

Nachrichten von der Gesellschaft der Wissenschaften zu Göttingen. Mathematisch-Physikalische Klasse. Neue Folge. Fachgruppe I. Nachrichten aus der Mathematik

Periodico di Matematiche

Philosophical Transactions of the Royal Society of London. Series A. Mathematical and Physical Sciences

Proceedings of the Cambridge Philosophical Society

Proceedings of the Imperial Academy

J. École Polytech.

J. Math. Pures Appl.

J. Reine Angew. Math.

J. Symbolic Logic

J. Fac. Sci. Imp. Univ. Tokyo. Sect. I

J. Indian Math. Soc.

J. London Math. Soc.

Neder. Akad.. Wetensch.

Math. Ann.

Math. Zeit.

Nachr. Ges. Wiss. Göttingen

Period. Math.

Philos. Trans. Roy. Soc. London. Ser. A.

Proc. Cambridge Philos. Soc.

Proc. Imp. Acad. Tokyo

Proceedings of the London Mathematical Proc. London Math. Soc. Society

Proceedings of the National Academy of Sci- Proc. Nat. Acad. Sci. U.S.A. ences of the United States of America

Proceedings of the Royal Society. Series A. Proc. Roy. Soc. London. Ser. A. Mathematical and Physical Sciences

The Quarterly Journal of Mathematics Oxford Series

Recueil Mathématique. Nouvelle Série

Rendiconti del Circolo Matematico di Palermo

Sitzungsberichte der Berliner Mathematischen Gesellschaft

Sitzungsberichte der Preussischen Akademie der Wissenschaften

The Tôhoku Mathematical Journal

Quart. J. Math. Oxford Ser.

Rec. Math. (Mat. Sbornik) N.S.

Rend. Circ. Mat. Palermo

Berlin Math. Ges. Sitzungsber.

Preuss. Akad. Wiss. Sitzungsber.

Tôhoku Math. J.

Transactions of the American Mathematica! Trans. Amer. Math. Soc. Society 


\section{APPENDIX C}

\section{A LIST OF SIGNS AND SPECIAL CHARACTERS AVAILABLE FOR THE BULLETIN AND TRANSACTIONS}

Lightface Greek- $\alpha \beta \gamma \ldots$ (all) A B $\Gamma \ldots$ (all).

$\star$ Lightface Greek Superiors- ${ }^{\Delta}$ and $\alpha \beta \gamma \ldots$ (all except 0 ).

$\star$ Lightface Greek Inferiors- $-\Delta \Delta \Sigma \Omega$ and $\alpha \beta \gamma \ldots$ (all except 0 ).

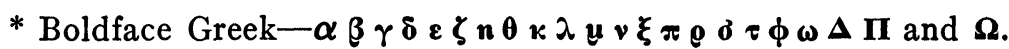

* Lightface German-â $\mathfrak{B} \mathfrak{S} \ldots$. (all) $\mathfrak{a} \mathfrak{b} \mathfrak{c} \ldots$. (all). No superiors or inferiors.

* Lightface dashed German- $\overline{\mathfrak{p}} \overline{\mathfrak{a}} \overline{\mathfrak{B}} \overline{\mathfrak{u}} \overline{\mathfrak{R}} \bar{\subseteq} \overline{\mathfrak{S}}$

* Boldface German $\longrightarrow \mathfrak{A} \mathfrak{B} \mathfrak{D}$

Script (special font) $\mathcal{A} B \mathcal{C} \ldots$ (all). No lower case manufactured.

${ }^{*}$ Hebrew $-N \wedge \mathbb{N} \Xi \sqsubset$ troublesome to use. No superior or inferior.

$\star$ Dashed Italics $-\bar{A} \bar{a} \bar{B} \bar{b} \bar{C} \bar{c} \ldots$ (all)

$\star$ Tilde Italics- $\tilde{A} \tilde{a} \tilde{e} \tilde{N} \tilde{n} \tilde{O} \tilde{r} \tilde{u} \tilde{y} \tilde{s} \tilde{M} \tilde{T} \tilde{B} \tilde{K}$

$\star$ Tilde Greek一 $\tilde{\alpha} \tilde{\boldsymbol{\epsilon}} \tilde{\eta} \tilde{\imath} \tilde{o} \tilde{v} \tilde{\omega}$

$\star$ Dashed Greek- $\bar{\alpha} \bar{\beta} \bar{\gamma} \bar{\delta} \bar{\eta} \bar{\theta} \bar{i} \bar{\mu} \bar{\nu} \bar{\rho} \bar{\omega} \bar{\lambda} \bar{\zeta} \bar{\sigma} \bar{\psi} \bar{\xi} \bar{\epsilon}$

$\star$ Dotted Italic-ia $\ddot{a} \dot{e} \ddot{e} \ddot{g} \ddot{i} \dot{m} \dot{n} \dot{q} \dot{r} \ddot{r} \dot{u} \ddot{u} \dot{x} \ddot{x} \dot{y} \ddot{y} \dot{z} \dot{z}$

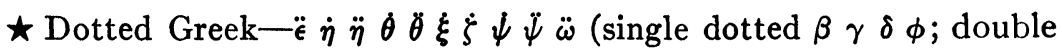
dotted $\gamma$ readily available).

$\star$ Superiors- $\dot{m} \dot{\boldsymbol{r}} \dot{\boldsymbol{s}} \mathbf{i} \dot{\mathbf{2}}$

$\star$ Inferiors- $\begin{array}{llllllllll}\dot{m} & \dot{n} & \bar{n} & \dot{r} & \dot{s} & \dot{x} & \dot{y} & \bar{z} & \mathbf{i} & \dot{2}\end{array}$

Signs-Superior- $+=\infty \pm$ ( ) [ ] ] , ' / I

Signs-Superior to Superior- -+

Signs-Inferior-_ $=+\infty \pm \neq$ ( ) [ ] . , / ,

Signs-Inferior to Inferior-+ - -

Special-Superior to Superior-1 $\begin{array}{lllll}1 & 2 & \mathbf{3} & 0 & \mathbf{m}\end{array}$

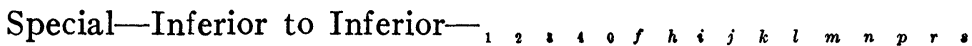

* Additional characters readily available at small cost.

$\star$ Matrices for additional characters are made upon special orders. Normally they would necessitate a delay of from four to eight weeks and average expense of $\$ 4.50$ per matrix. However, because of wartime conditions delivery dates are uncertain and prices subject to change without notice. 


\begin{tabular}{|c|c|c|c|c|c|c|c|c|c|}
\hline No. & 6 & 8 & 10 & 12 & No. & 6 & 8 & 10 & $12^{4}$ \\
\hline 1. - & $\checkmark$ & $v$ & $\checkmark$ & $\sqrt{ }$ & 40. V & & $\checkmark$ & $\checkmark$ & \\
\hline 2. $\times$ & $\sqrt{ }$ & $\sqrt{ }$ & $\checkmark$ & $\sqrt{ }$ & 41. כ & & & $\checkmark$ & \\
\hline 3. 8 & & $\sqrt{ }$ & & & 42. c & & & $\checkmark$ & \\
\hline 4. $\div$ & $\checkmark$ & $\checkmark$ & $\checkmark$ & $\checkmark$ & 43. † & & & $\checkmark$ & \\
\hline 5. + & $\checkmark$ & $\checkmark$ & $\checkmark$ & $\checkmark$ & 44. u & $\checkmark$ & & & \\
\hline 6. + & & & $\sqrt{ }$ & & 45. ก & & $\sqrt{ }$ & & \\
\hline 7. \pm & & $\checkmark$ & & & 46. $\quad \therefore$ & & & $\checkmark$ & \\
\hline 8. \pm & $\sqrt{ }$ & $\checkmark$ & $\sqrt{ }$ & $\checkmark$ & 47. $\triangle$ & & & $\checkmark$ & \\
\hline 9. $\mp$ & & & $\checkmark$ & & 48. 今 & & & $\checkmark$ & \\
\hline 10. $=$ & $\checkmark$ & $\checkmark$ & $\checkmark$ & $\sqrt{ }$ & 49. \ & & $\sqrt{ }$ & & \\
\hline 11. $=$ & & $\checkmark$ & $\sqrt{ }$ & & $50 . \quad \Delta$ & & $\sqrt{ }$ & & \\
\hline 12. $\doteq$ & $\sqrt{ }$ & & $\sqrt{ }$ & & 51. $\angle$ & & $\sqrt{ }$ & $\checkmark$ & \\
\hline 13. $\cong$ & $\sqrt{ }$ & & $\checkmark$ & & 52. ८ & & & $\checkmark$ & \\
\hline 14. $\cong$ & & $\checkmark$ & & & 53. $\square$ & & & $\checkmark$ & \\
\hline 15. $\neq$ & $\sqrt{ }$ & $\checkmark$ & $\checkmark$ & & 54. 凹 & & $\sqrt{ }$ & & \\
\hline 16. $\neq$ & & $\checkmark$ & $\checkmark$ & & 55. & & $\sqrt{ }$ & & \\
\hline 17. $\equiv$ & & $\checkmark$ & $\checkmark$ & & 56. $\square$ & & & $\checkmark$ & \\
\hline 18. $\not \equiv$ & & $\sqrt{ }$ & $\checkmark$ & & 57. ब & & & $\checkmark$ & \\
\hline 19. $\neq$ & & & $\sqrt{ }$ & & 58. $\square$ & & & $\checkmark$ & \\
\hline 20. $\infty$ & $\sqrt{ }$ & $\checkmark$ & $\checkmark$ & & 59. s & & & $\checkmark$ & \\
\hline 21. $\sim$ & & $\checkmark$ & $\checkmark$ & & $60 . \diamond$ & & $\sqrt{ }$ & & \\
\hline 22. $\propto$ & & & $\checkmark$ & & 61. $\bigcirc$ & & $\sqrt{ }$ & $\checkmark$ & \\
\hline 23. $\approx$ & & $\checkmark$ & $\checkmark$ & & 62. $\odot$ & & $\checkmark$ & $\checkmark$ & \\
\hline 24. $\sim$ & & $\checkmark$ & $\checkmark$ & & 63. (s) & & & $\checkmark$ & \\
\hline 25. $\sim$ & & $\sqrt{ }$ & & & 64. ఠ & & $\sqrt{ }$ & & \\
\hline 26. $\approx$ & & & $\sqrt{ }$ & & 65. $\odot$ & & $\sqrt{ }$ & & \\
\hline $27 .<$ & $\checkmark$ & $\checkmark$ & $\checkmark$ & & 66. $\theta$ & & & $\checkmark$ & \\
\hline 28. $\lessgtr$ & & & $\checkmark$ & & 67. $\oplus$ & & & $\checkmark$ & \\
\hline 29. $>$ & $\checkmark$ & $\checkmark$ & $\checkmark$ & & 68. $\otimes$ & & $\checkmark$ & $\checkmark$ & \\
\hline 30. $\gtrless$ & & & $\checkmark$ & & 69. $\varnothing$ & & & $\checkmark$ & \\
\hline 31. $\leqq$ & $\checkmark$ & $\checkmark$ & $\checkmark$ & & 70. ळ & & & $\checkmark$ & \\
\hline 32. $\geqq$ & $\checkmark$ & $\checkmark$ & $\sqrt{ }$ & & 71. $\nabla$ & & & $\checkmark$ & \\
\hline 33. $\ngtr$ & & $\checkmark$ & $\checkmark$ & $\checkmark$ & 72. $\rightarrow$ & $\checkmark$ & $\checkmark$ & $\checkmark$ & \\
\hline $34 . \nless$ & & $\checkmark$ & & & $73 . \leftarrow$ & & $\checkmark$ & $\checkmark$ & \\
\hline 35. $\wedge$ & & $\sqrt{ }$ & $\sqrt{ }$ & & 74. $\rightarrow$ & $\sqrt{ }$ & $\sqrt{ }$ & $\checkmark$ & \\
\hline 36. $\vee$ & & $\checkmark$ & $\checkmark$ & & 75. $\rightleftarrows$ & & & $\checkmark$ & \\
\hline 37. $\pi$ & & $\checkmark$ & & & 76. $\leftrightarrows$ & & & & $\checkmark$ \\
\hline $38 . \ll$ & & & $\checkmark$ & & 77. & & $\checkmark$ & & \\
\hline 39. $\Varangle$ & & & $\checkmark$ & & 78. $\rightleftarrows$ & & & $\checkmark$ & \\
\hline
\end{tabular}

${ }^{4}$ These figures refer to the size of type. The text of Bulletin and Transactions is 10 point type and footnotes 8 point type. 


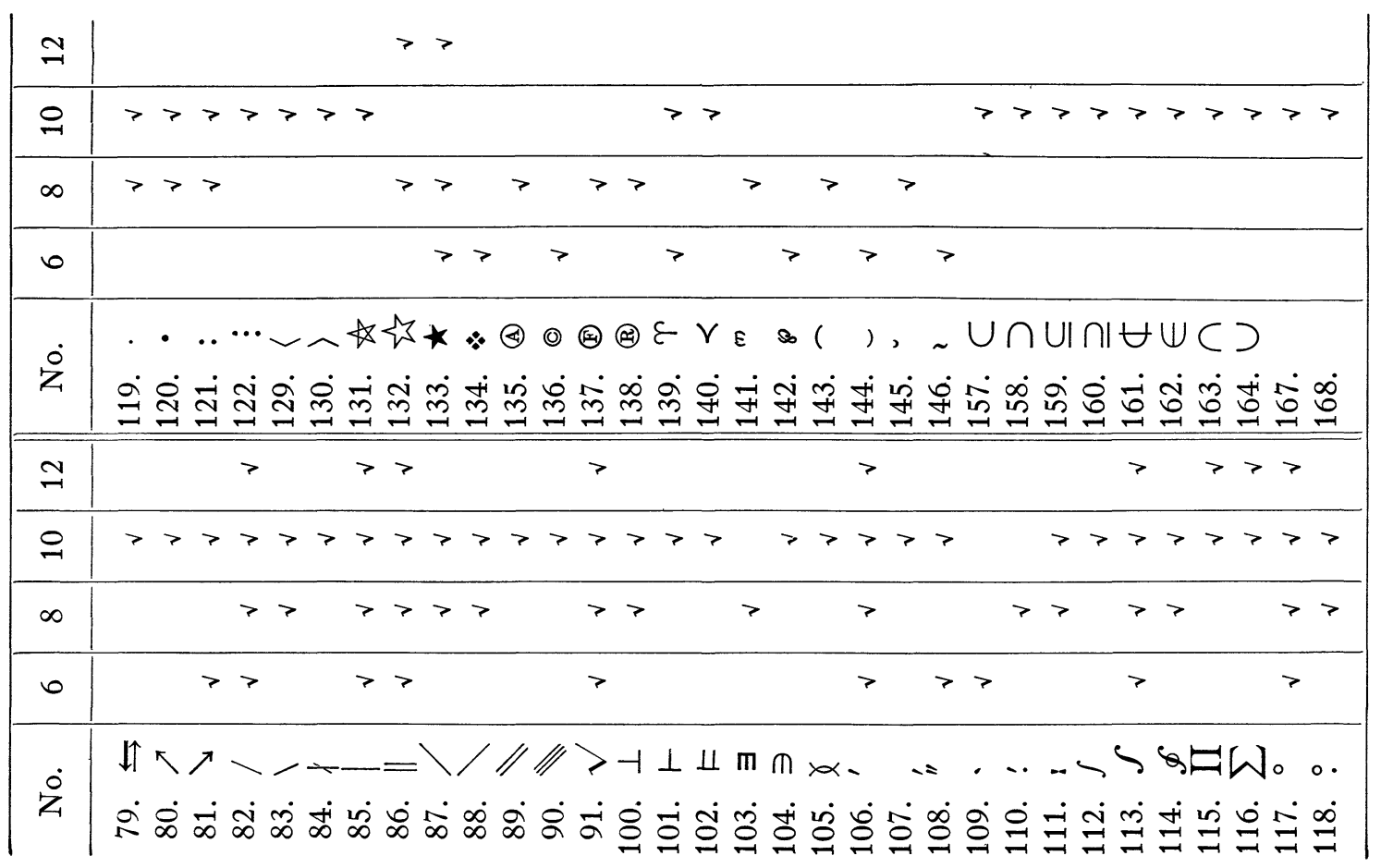

